\title{
Nivel de conocimiento sobre factores de riesgo cardiovascular en una comunidad de Naguanagua, Venezuela
}

The level of knowledge concerning cardiovascular risk factors in people living in Naguanagua, Venezuela

\author{
Marvin Querales ${ }^{1}$, Nelina Ruiz ${ }^{2}$, Susan Rojas ${ }^{1}$ y Milagros Espinoza ${ }^{3}$
}

\begin{abstract}
1 Departamento de Bioquímica. Escuela de Ciencias Biomédicas y Tecnológicas. Facultad de Ciencias de la Salud. Universidad de Carabobo. Valencia, Venezuela. E-mail: marvinquerales@hotmail.com susanrojas272@hotmail.com

2 Departamento de Morfofisiopatología. Escuela de Bioanálisis. Facultad de Ciencias de la Salud. Universidad de Carabobo. Valencia, Venezuela. E-mail: nelinaruiz@yahoo.com

3 Departamento de Investigación y Desarrollo Profesional. Escuela de Bioanálisis. Facultad de Ciencias de la Salud. Universidad de Carabobo. Naguanagua, Venezuela. E-mail: eszami@hotmail.com
\end{abstract}

Recibido 7 Febrero 2011/Enviado para Modificación 23 Octubre 2011/Aceptado 30 Octubre 2011

\section{RESUMEN}

Objetivo Evaluar el nivel de conocimiento de los factores de riesgo cardiovascular y asociarlo con alteraciones de los marcadores clínicos, antropométricos y bioquímicos de riesgo cardiovascular en una comunidad del Municipio Naguanagua, Venezuela.

Métodos Se evaluaron 205 pacientes con edades comprendidas entre 18 y 77 años, a los cuales se les determinó peso, talla, circunferencia abdominal, presión arterial, glicemia y perfil lipídico. Se aplicó una encuesta para medir el nivel de conocimiento de los factores de riesgo cardiovascular.

Resultados Se evidenció una alta frecuencia de obesidad abdominal (67\%) sobrepeso y obesidad (38 \%) e Hipertensión (26\%). Casi $60 \%$ de los participantes afirmó conocer los factores de riesgo cardiovascular, pero sólo 14,7\% reconocieron los once factores que se consideraron para definir el nivel de conocimiento. Más de la mitad de los participantes mostraron un bajo nivel de conocimiento de factores de riesgo cardiovasculares cuyos niveles promedio de colesterol total, LDL colesterol y triglicéridos fueron significativamente menores a los del grupo de alto nivel de conocimiento.

Conclusión Se encontró un porcentaje elevado de individuos con bajo nivel de conocimiento de los factores de riesgo cardiovascular y una frecuencia de alteraciones de los parámetros clínicos, antropométricos y bioquímicos mayor entre los participantes con alto nivel de conocimiento, confirmando la necesidad de ejecutar estrategias que no sólo eleven el nivel de conocimiento de las comunidades venezolanas, sino también motiven efectivamente a la adopción de un estilo de vida asociado a la reducción de los factores de riesgo cardiovascular y al autocuidado de la salud. 
Palabras Clave: Conocimiento, factores de riesgo, enfermedad cardiovascular (fuente: DeCS, BIREME).

\section{ABSTRACT}

Objective Associating the level of awareness concerning cardiovascular risk factors related to changes in clinical, anthropometric and biochemical markers regarding cardiovascular risk in people living in Naguanagua, Venezuela.

Method 205 patients aged 18 to 77 years were evaluated; weight, height, waist circumference, blood pressure, glucose and serum lipid profile were measured. A survey assessed the patients' level of awareness regarding cardiovascular risk factors.

Results A high frequency of abdominal obesity (67\%), being overweight and obese $(38 \%)$ and suffering from hypertension (26\%) was observed. Nearly $60 \%$ of respondents claimed to know about cardiovascular risk factors, but only $14.7 \%$ of them recognized the eleven factors considered to define such level of knowledge. More than half of the participants showed a low level of awareness concerning cardiovascular risk factors; average levels of total cholesterol, cholesterol LDL and triglycerides were significantly lower in the group having a high level of knowledge concerning cardiovascular risk factors.

Conclusions Extremely low levels of awareness regarding cardiovascular risk factor prevalence and high frequency concerning changes in clinical, anthropometric and biochemical markers for cardiovascular risk were higher in the group having a high level of knowledge of cardiovascular risk factors, thereby confirming the need for implementing strategies for raising Venezuelan communities' level of knowledge regarding cardiovascular risk factors and also encouraging them to adopt a lifestyle associated with reduced cardiovascular risk factors and self-care.

Key Words: Knowledge, awareness, risk factor, cardiovascular disease (source: $\mathrm{MeSH}, \mathrm{NLM}$ ).

$\mathrm{L}$ a incidencia de las enfermedades cardiovasculares (ECV) ha alcanzado cifras alarmantes en muchas regiones del mundo $(1,2)$, incluyendo Venezuela (3). Los distintos programas de prevención de las ECV están focalizados en reducir los factores de riesgo modificables a través de cambios en el estilo de vida. Existen modelos que proponen que el conocimiento de los factores de riesgo cardiovascular por parte del individuo es una condición necesaria para producir modificaciones en la conducta (4), aunque, el conocimiento por sí sólo no es suficiente para promover un cambio en la actitud, ya que la transición entre la motivación y la acción pudiera verse obstaculizada por diversas condiciones (5). En diferentes países se ha evidenciado un bajo grado de identificación de los factores de riesgo cardiovascular $(6,7)$. 
En Venezuela, en una comunidad del Estado Carabobo, un estudio previo demostró que aproximadamente $30 \%$ de los individuos no logró identificar alguno de los factores de riesgo para hipertensión arterial (HTA) y enfermedad cardiaca coronaria (8), resultados que pudieran estar asociados con la alta prevalencia de factores de riesgo cardiometabólico encontrada en la misma comunidad (9). De evidenciarse esta relación, se tendría un basamento más sólido para el diseño e implementación de estrategias educativas de salud para un abordaje efectivo de esta problemática.

El objetivo de la investigación fue evaluar el nivel de conocimiento de los factores de riesgo cardiovascular y asociarlo con la frecuencia de alteración de los principales indicadores clínicos, antropométricos y bioquímicos, en una comunidad del Municipio Naguanagua, Venezuela.

\section{MATERIALES Y MÉTODOS}

Estudio descriptivo y correlacional que incluyó 205 pacientes que participaron en jornadas de despistaje de HTA en el Ambulatorio Docente-Asistencial "Nuestra Señora de la Luz", Municipio Naguanagua, Estado Carabobo, Venezuela, durante los meses Febrero-Diciembre de los años 2008-2009. El estudio contó con el aval del comité de ética del centro de salud y todos los participantes firmaron consentimiento informado (10).

Los participantes acudieron luego de 12 horas de ayuno, sin haber ingerido alcohol 24 horas antes de la toma de muestra. Inicialmente, se aplicó una encuesta para obtener datos personales, socioeconómicos, demográficos, antecedentes personales y familiares en primer grado de consanguinidad de diabetes mellitus (DM) y de ECV; hábitos tabáquico y alcohólico; actividad física y tratamiento farmacológico. El estrato socioeconómico (ESE) se estableció por el método de Graffar modificado (11). Se definió fumador aquel participante que fumaba al momento de la evaluación o abandonó el hábito dentro de los cinco años previos a ésta (12) y como actividad física regular, si el participante realizaba 30 minutos de caminata por lo menos 5 días a la semana (13).

La segunda parte de la encuesta, diseñada "ah hoc" fue validada a través de una prueba piloto y sometida a juicio de expertos. Esta permitió conocer el nivel de conocimiento de los participantes en cuanto a los factores de riesgo relacionados con ECV. Fueron once los factores de riesgo encuestados: colesterol elevado, grasa en la dieta, consumo de sal, diabetes, estrés, fumar, 
HTA, sedentarismo, sobrepeso y obesidad, consumo excesivo de alcohol y café. Para definir el nivel de conocimiento se obtuvo la distribución percentilar del número de factores de riesgo correctamente identificados por los individuos estudiados, considerándose que un participante tenia nivel de conocimiento bajo cuando identificó hasta tres (percentil 25) de los factores de riesgo encuestados; intermedio entre cuatro y ocho factores; y alto cuando identificó nueve (percentil 75) o más factores. La encuesta también incluyó un listado de alimentos para identificar aquellos que pudieran estar relacionados o producir ECV y a los que pudieran prevenirlas.

Personal entrenado realizó mediciones de peso, talla, circunferencia de cintura (CC) y presión arterial siguiendo los protocolos recomendados $(14,15)$. Para pesar se utilizó una balanza (HealthMeter) previamente calibrada (precisión $=0,1 \mathrm{~g}$ ); la talla se midió con ayuda del estadiómetro de la balanza (precisión $=1 \mathrm{~mm}$ ); la $\mathrm{CC}$ se determinó con una cinta métrica no extensible (precisión $=1 \mathrm{~mm}$ ). Las mediciones antropométricas se realizaron sin zapatos y con ropa mínima. Se calculó el índice de masa corporal (IMC) mediante la fórmula: peso $(\mathrm{kg}) /\left(\right.$ talla $^{2}(\mathrm{~m})$.

Se extrajo una muestra de sangre, por punción venosa en el pliegue del codo, para determinar parámetros como: glucosa, colesterol total y triglicéridos (método enzimático-colorimétrico); colesterol unido a la lipoproteína de alta densidad (HDLc) después de precipitación con fosfotungstato. El colesterol unido a la lipoproteína de baja densidad (LDLc) se calculó a través de la fórmula de Fridewald (16). Para definir sobrepeso/obesidad según IMC obesidad abdominal, HTA, DM y alteraciones del perfil lipídico se emplearon los criterios establecidos internacionalmente $(15,17-19)$.

Para el análisis de los datos se utilizó el programa estadístico SPSS. Las variables cuantitativas se expresaron como media aritmética y desviación estándar, mientras que las cualitativas como porcentajes. Para comparar las variables continuas según nivel de conocimiento se empleó ANOVA. El test chi cuadrado $\left(\chi^{2}\right)$ permitió asociar la frecuencia de alteración de los indicadores con el nivel de conocimiento. Se consideró significancia estadística cuando $\mathrm{p}<0,05$.

\section{RESULTADOS}

Se evaluaron 205 pacientes con edades entre 18 y 77 años (47,4 $\pm 13,0$ años) de los cuales $21 \%$ pertenecían al género masculino y $79 \%$ al femenino. Las 
características del grupo en estudio se muestran en la Tabla 1; fundamentalmente estuvo integrado por individuos ubicados en los ESE más bajos. Se observó un porcentaje bajo de analfabetismo mientras que un poco más del tercio de individuos solamente sabía leer y escribir, cursó sólo primaria o hasta noveno año de secundaria pero sin lograr aprobarlo. Más de la mitad de los pacientes encuestados presentaron antecedentes de HTA, siendo la prevalencia de la misma de $25,9 \%$.

Tabla 1. Características de la muestra según género (\%)

\begin{tabular}{|c|c|c|c|}
\hline & $\begin{array}{l}\text { Masculino } \\
(n=43)\end{array}$ & $\begin{array}{l}\text { Femenino } \\
(n=162)\end{array}$ & $\begin{array}{l}\text { Grupo Total } \\
(n=205)\end{array}$ \\
\hline \multicolumn{4}{|l|}{ Grado de Instrucción } \\
\hline Analfabeto & 4,7 & 6,8 & 6,3 \\
\hline Sólo sabe leer y escribir, cursó solo primaria & 32,6 & 37,7 & 36,6 \\
\hline $\begin{array}{l}\text { Secundaria incompleta (mínimo noveno año de } \\
\text { secundaria aprobado) }\end{array}$ & 18,6 & 25,3 & 23,9 \\
\hline Secundaria completa, Técnico medio o TSU & 39,5 & 24,1 & 27,3 \\
\hline Enseñanza universitaria & 4,7 & 5,6 & 5,4 \\
\hline \multicolumn{4}{|l|}{ Estrato socioeconómico } \\
\hline Estrato III & 16,3 & 21,7 & 20,2 \\
\hline Estrato IV & 65,1 & 65,8 & 66,0 \\
\hline Estrato V & 18,6 & 12,4 & 13,8 \\
\hline \multicolumn{4}{|l|}{ Estado Civil } \\
\hline Soltero* & 39,5 & 59,3 & 55,1 \\
\hline Casado & 53,5 & 33,9 & 38,1 \\
\hline Divorciado & 7,0 & 5,6 & 5,9 \\
\hline \multicolumn{4}{|l|}{ Antecedente personal de Hipertensión Arterial } \\
\hline \multicolumn{4}{|l|}{ Antecedente personal de Diabetes Mellitus } \\
\hline autoreportado & 25,6 & 24,1 & 24,4 \\
\hline Antecedente personal de $\mathrm{ECl}$ autoreportado & 23,3 & 26,5 & 25,9 \\
\hline Sobrepeso & 53,5 & 33,9 & 38,1 \\
\hline Obesidad & 27,9 & 39,5 & 37,1 \\
\hline Obesidad Abdominal & 51,2 & 71,6 & 67,3 \\
\hline Hipercolesterolemia & 25,6 & 50,6 & 45,4 \\
\hline LDLc elevada & 11,6 & 22,8 & 20,5 \\
\hline HDLc baja & 46,5 & 88,3 & 79,5 \\
\hline Hipertrigliceridemia & 41,9 & 35,8 & 37,1 \\
\hline \multicolumn{4}{|l|}{ Hábito Tabáquico } \\
\hline Fumador & 23,3 & 17,9 & 19,0 \\
\hline Ex fumador & 16,3 & 20,4 & 19,5 \\
\hline No fumador & 74,4 & 75,3 & 75,1 \\
\hline Hipertensión Arterial & 30,2 & 24,7 & 25,9 \\
\hline Tratamiento antihipertensivo & 16,3 & 23,5 & 21,9 \\
\hline Diabetes mellitus & 9,3 & 5,6 & 6,3 \\
\hline $\begin{array}{l}\text { Individuos que reportaron consumo de bebidas } \\
\text { alcohólicas }\end{array}$ & 62,8 & 40,1 & 44,9 \\
\hline Individuos que reportaron actividad física regular & 7,0 & 21,6 & 18,5 \\
\hline
\end{tabular}


Se evidenció una alta frecuencia de sobrepeso y obesidad, encontrándose que más del $50 \%$ de los hombres presentó exceso de peso. La obesidad abdominal mostró una importante frecuencia sobre todo en mujeres, alcanzando cifras de 71,6\%. La disminución de los niveles de HDLc fue la dislipidemia más prevalente. Cerca de la mitad de la muestra reportó consumo de bebidas alcohólicas y un porcentaje menor al 20 \% refirió realizar actividad física regular y hábito tabáquico.

Tabla 2. Conocimiento de los factores de riesgo relacionados con enfermedades cardiovasculares en general

\begin{tabular}{|c|c|c|}
\hline Pregunta & \multicolumn{2}{|c|}{$\begin{array}{l}\text { Frecuencia respuestas afirmativas } \\
\qquad \%(n=205)\end{array}$} \\
\hline $\begin{array}{l}\text { ¿Conoce usted los factores de riesgo } \\
\text { para enfermedad cardiovascular? }\end{array}$ & \multicolumn{2}{|l|}{$59,0(121)$} \\
\hline $\begin{array}{l}\text { ¿Cuáles considera factores de riesgo } \\
\text { para enfermedad cardiovascular? }\end{array}$ & $\begin{array}{l}\text { Colesterol elevado } \\
\text { Grasa en la dieta } \\
\text { Mala alimentación } \\
\text { Sal } \\
\text { Diabetes } \\
\text { Edad } \\
\text { Estrés } \\
\text { Fumar } \\
\text { Herencia } \\
\text { Hipertensión } \\
\text { Sedentarismo } \\
\text { Género } \\
\text { Sobrepeso y Obesidad } \\
\text { Consumo excesivo de alcohol } \\
\text { Consumo excesivo de café }\end{array}$ & $\begin{array}{c}40,9(84) \\
53,7(110) \\
15,3(15)^{\dagger} \\
41,9(86) \\
34,2(70) \\
5,1(5)^{\dagger} \\
45,4(93) \\
49,3(101) \\
9,2(9)^{\dagger} \\
44,9(92) \\
40,5(83) \\
3,1(3)^{\dagger} \\
42,4(87) \\
46,3(95) \\
36,1(74)\end{array}$ \\
\hline $\begin{array}{c}\text { ¿Qué alimentos cree Ud. que pudieran } \\
\text { estar relacionados o producir } \\
\text { enfermedad cardiovascular? }\end{array}$ & $\begin{array}{l}\text { Vegetales } \\
\text { Frutas } \\
\text { Fibras } \\
\text { Carnes } \\
\text { Grasa } \\
\text { Sal } \\
\text { Alcohol } \\
\text { Azúcares }\end{array}$ & $\begin{array}{c}1,1(1)^{\dagger} \\
0^{\dagger} \\
1,1(1)^{\dagger} \\
32,7(32)^{\dagger} \\
80,6(79)^{\dagger} \\
28,6(28)^{\dagger} \\
32,7(32)^{\dagger} \\
21,4(21)^{\dagger}\end{array}$ \\
\hline $\begin{array}{c}\text { ¿Qué alimentos cree Ud. que pudieran } \\
\text { prevenir las enfermedades } \\
\text { cardiovasculares? }\end{array}$ & $\begin{array}{l}\text { Vegetales } \\
\text { Frutas } \\
\text { Fibras } \\
\text { Carnes } \\
\text { Grasa } \\
\text { Sal } \\
\text { Alcohol } \\
\text { Azúcares }\end{array}$ & $\begin{array}{c}61,2(60)^{\dagger} \\
51,0(50)^{\dagger} \\
25,5(25)^{\dagger} \\
4,1(4)^{\dagger} \\
6,1(6)^{\dagger} \\
3,1(3)^{\dagger} \\
2,0(2)^{\dagger} \\
3,1(3)^{\dagger}\end{array}$ \\
\hline
\end{tabular}

Los resultados de la encuesta sobre el conocimiento de los factores de riesgo para ECV se muestran en la Tabla 2. Aproximadamente la mitad de los participantes, afirmaron conocer los factores de riesgo cardiovascular, siendo cuatro la mediana del número de factores reconocidos por la muestra 
estudiada. Sólo 14,7 \% reconocieron los once factores de riesgo estudiados. El factor identificado con mayor frecuencia fue la grasa en la dieta $(53,7 \%)$, seguido del hábito tabáquico (49,3\%), consumo excesivo de alcohol (46,3\%) y estrés $(45,4 \%)$. La mayoría identificó a la grasa $(80,6 \%)$, la carne $(32,7 \%)$ y el alcohol $(32,7 \%)$ como alimentos que pudieran estar relacionados con la ECV. Por su parte, los vegetales $(61,2 \%)$ y frutas $(51,0 \%)$ fueron los más frecuentemente reconocidos como alimentos que pueden prevenir dichas enfermedades. La identificación de los factores de riesgo evaluados, no se asoció significativamente al género.

Figura 1. Individuos estudiados según nivel de conocimiento de factores de riesgo cardiovascular

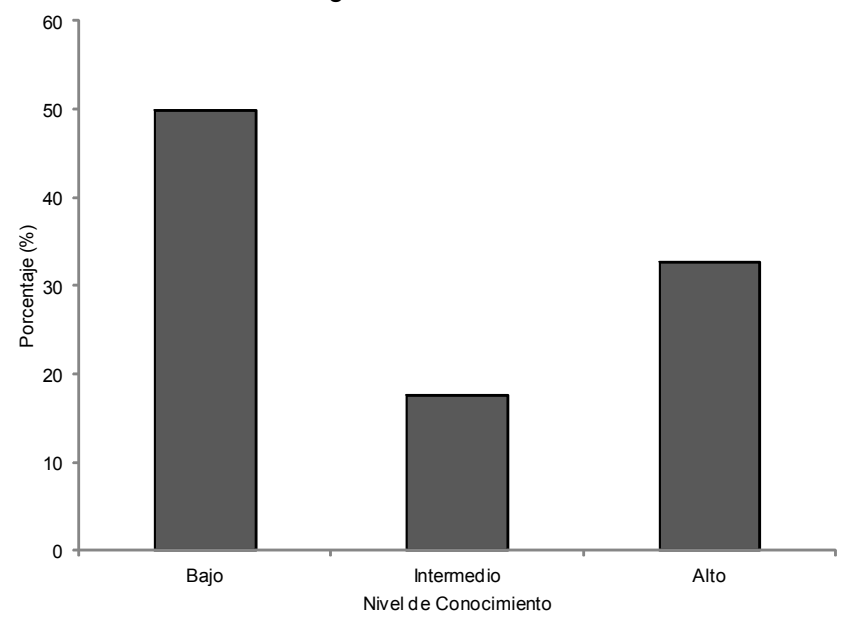

Más de mitad de los participantes mostraron un bajo nivel de conocimiento de factores de riesgo cardiovasculares (59,0 \%) (Figura 1). Aunque no se alcanzaron diferencias significativas según género, el mayor porcentaje de los hombres mostró un nivel de conocimiento bajo e intermedio, mientras que aproximadamente la mitad de las mujeres presentó un nivel intermedio o alto de conocimiento (Figura 2).

Los niveles de colesterol total y LDLc fueron significativamente más elevados en el grupo de alto nivel de conocimiento de factores de riesgo cardiovascular, en comparación con el de bajo conocimiento (Tabla 3). Resultado similar se observó para las concentraciones de triglicéridos. 
Figura 2. Individuos estudiados por género según nivel de conocimiento de factores de riesgo cardiovascular

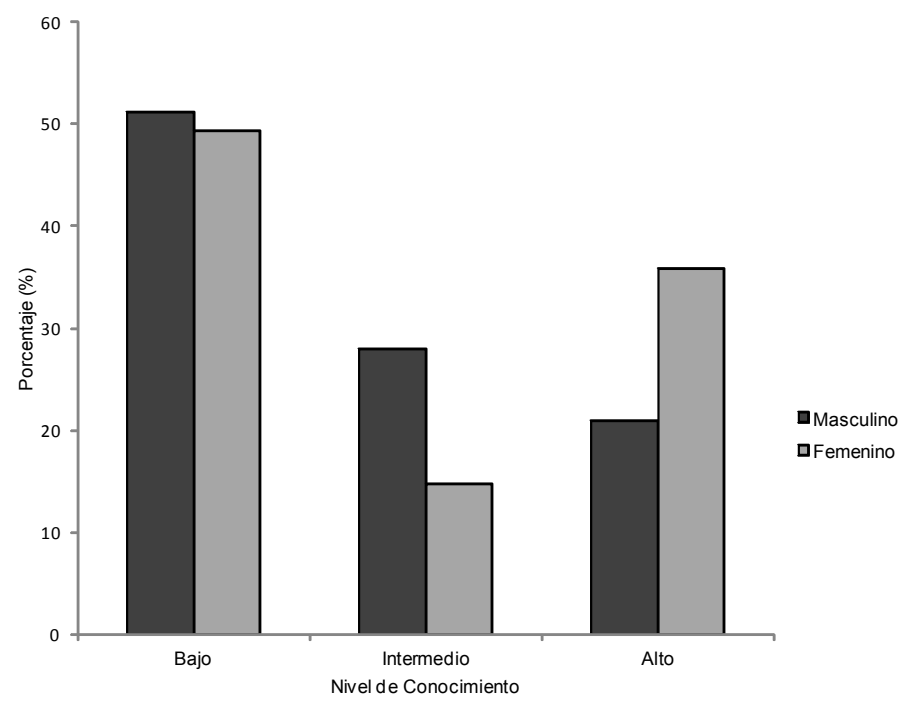

Tabla 3. Indicadores clínicos, antropométricos y bioquímicos evaluados en el grupo total y de acuerdo al nivel de conocimiento de los factores de riesgo cardiovascular

\begin{tabular}{lccccc}
\hline \multicolumn{1}{c}{ Indicadores } & $\begin{array}{c}\text { Grupo Total } \\
(\mathrm{n}=205)\end{array}$ & $\begin{array}{c}\text { Bajo } \\
(\mathrm{n}=102)\end{array}$ & $\begin{array}{c}\text { Nivel de Conocimiento* } \\
\text { Intermedio } \\
(\mathrm{n}=36)\end{array}$ & $\begin{array}{c}\text { Alto } \\
(\mathrm{n}=67)\end{array}$ & $\mathrm{p}$ \\
\hline PAS $(\mathrm{mmHg})$ & $123,4 \pm 17,6$ & $125,1 \pm 16,5$ & $123,6 \pm 18,4$ & $120,8 \pm 18,7$ & 0,3090 \\
PAD $(\mathrm{mmHg})$ & $79,1 \pm 12,9$ & $79,7 \pm 12,9$ & $79,9 \pm 14,6$ & $77,7 \pm 12,2$ & 0,5813 \\
IMC $\left(\mathrm{kg} / \mathrm{m}^{2}\right)$ & $28,8 \pm 5,4$ & $28,8 \pm 5,3$ & $29,1 \pm 6,1$ & $28,6 \pm 5,3$ & 0,9131 \\
CA $(\mathrm{cm})$ & $95,8 \pm 12,8$ & $96,4 \pm 10,9$ & $96,9 \pm 16,3$ & $94,5 \pm 13,3$ & 0,5636 \\
Glicemia $(\mathrm{mg} / \mathrm{dL})$ & $94,1 \pm 37,5$ & $92,1 \pm 43,3$ & $91,2 \pm 25,0$ & $98,9 \pm 33,4$ & 0,4521 \\
Col Total $(\mathrm{mg} / \mathrm{dL})$ & $196,1 \pm 45,9$ & $185,4 \pm 50,3$ & $193,3 \pm 43,4$ & $213,7 \pm 34,2$ & 0,000 \\
LDLc $(\mathrm{mg} / \mathrm{dL})$ & $125,5 \pm 41,8$ & $116,1 \pm 45,5$ & $124,9 \pm 38,6$ & $140,1 \pm 33,2$ & 0,001 \\
HDLc $(\mathrm{mg} / \mathrm{dL})$ & $40,7 \pm 6,2$ & $41,0 \pm 6,9$ & $41,4 \pm 6,3$ & $39,9 \pm 4,9$ & 0,3937 \\
TGL $(\mathrm{mg} / \mathrm{dL})$ & $155,7 \pm 86,0$ & $145,2 \pm 79,4$ & $136,8 \pm 57,9$ & $181,6 \pm 101,9$ & 0,008 \\
\hline PAS: Presión arterial sistólica; PAD: Presión arterial diastólica; mmHg: milimetros de mercurio; IMC: índice \\
de masa corporal; CA: circunferencia abdominal; Col Total: Colesterol Total; TG: Triglicéridos. * Nivel de \\
conocimiento Bajo=hasta 3 factores de riesgo identificados; Intermedio=entre 4 y 8 factores identificados; \\
Alto=9 o más factores identificados. Análisis de Varianza de un Factor.
\end{tabular}

En el grupo total, las frecuencias de hipercolesterolemia y HDLc disminuido, fueron significativamente más elevadas entre los individuos que mostraron un alto nivel de conocimiento de los factores de riesgo cardiovascular (Tabla 4). Al realizar el mismo análisis por género, el número de hombres con obesidad abdominal, hipercolesterolemia, aumento de LDLc 
e hipertrigliceridemia fue significativamente más elevado entre aquellos que mostraron un alto nivel de conocimiento de factores de riesgo cardiovascular. En las mujeres no se encontraron asociaciones significativas entre las alteraciones de los parámetros estudiados y el nivel de conocimiento de los factores de riesgo cardiovascular.

Tabla 4. Frecuencia de alteraciones de indicadores clínicos, antropométricos y bioquímicos según nivel de conocimiento de los factores de riesgo cardiovascular en el grupo total

\begin{tabular}{lccccc}
\hline \multicolumn{1}{c}{ Indicadores } & $\begin{array}{c}\text { Grupo } \\
\text { Total } \\
(\mathrm{n}=205)\end{array}$ & $\begin{array}{c}\text { Bajo } \\
(\mathrm{n}=102)\end{array}$ & $\begin{array}{c}\text { Nivel de Conocimiento } \\
\text { Intermedio } \\
(\mathrm{n}=36)\end{array}$ & $\begin{array}{c}\text { Alto } \\
(\mathrm{n}=67)\end{array}$ & $\mathrm{p}$ \\
\hline Sobrepeso & 38,1 & 38,2 & 36,1 & 40,3 & 0,9134 \\
Obesidad & 37,1 & 37,3 & 41,7 & 34,3 & 0,7621 \\
Obesidad Abdominal & 67,3 & 66,7 & 58,3 & 70,2 & 0,4786 \\
Hipercolesterolemia & 45,4 & 36,3 & 44,4 & 59,7 & 0,0113 \\
LDLc elevada & 20,5 & 14,7 & 22,2 & 28,4 & 0,0950 \\
HDLc baja & 79,5 & 78,4 & 66,7 & 88,1 & 0,0347 \\
Hipertrigliceridemia & 37,1 & 29,4 & 36,1 & 49,3 & 0,3578 \\
\hline HDLc: Colesterol unido a la lipoproteína de alta densidad; LDLc: Colesterol unido a la lipoproteína de \\
baja densidad. Nivel de conocimiento Bajo= hasta 3 factores de riesgo identificados; Intermedio= \\
entre 4 y 8 factores identificados; Alto= 9 o más factores identificados. Test chi cuadrado (X').
\end{tabular}

\section{DISCUSIÓN}

En el presente estudio se obtuvo una prevalencia de HTA de alrededor $26 \%$, resultado consistente con las cifras reportadas en otras investigaciones (20). También una proporción importante de individuos presentó sobrepeso u obesidad y obesidad abdominal, hallazgo similar se ha encontrado en trabajadores colombianos (21), lo cual pudiera guardar relación con hábitos alimentarios inadecuados y la inactividad física aquí evidenciada. Cabe destacar que el aumento de la CC profundiza los efectos metabólicos del exceso de peso, tales como resistencia insulínica, intolerancia a la glucosa o DM, HTA, desórdenes hemostáticos, hiperandrogenismo e inflamación (22), condiciones todas asociadas a mayor riesgo cardiovascular.

Casi $60 \%$ de los participantes afirmó conocer los factores de riesgo cardiovascular evaluados, lo cual coincide con lo reportado por Saeed (7), en la India. No obstante, al explorar el conocimiento específico de cada uno de los factores, un porcentaje similar no logró identificar más de tres factores de riesgo para ECV. Este resultado coincide con otros reportes internacionales $(23,24)$ y pudiera explicarse con base a que la mayoría de los participantes tenían bajo ESE y mínimo nivel de instrucción. Investigaciones previas han evidenciado que el grado de conocimiento de los factores de riesgo cardiovascular está fuertemente asociado al ESE (25). 
En una investigación realizada en Pakistán los individuos estudiados identificaron como factores de riesgo al estrés, HTA, sobrepeso y sedentarismo (26), mientras que en el presente trabajo los factores identificados con mayor frecuencia fueron la grasa dietaria y el hábito tabáquico, en semejanza a lo reportado en Canadá y en otro estudio paquistaní $(6,24)$. Dicho patrón de conocimiento pudiera deberse a que las campañas de prevención para ECV profundizan en la erradicación del hábito tabáquico y en la disminución del consumo de grasas saturadas. En este sentido, en este estudio una gran proporción $(80 \%)$ identificó las grasas y su relación con las ECV y también asoció el consumo de vegetales y frutas su prevención. No obstante, existieron alimentos como la sal y los azúcares, implicados en la predisposición a HTA y enfermedades coronarias, que no fueron identificados así como tampoco fue reconocida la fibra, como beneficiosa. Por lo anterior, es notoria la necesidad de implementar programas de educación nutricional que orienten sobre las ventajas y desventajas de consumir ciertos alimentos como estrategia para incentivar hábitos saludables de alimentación.

Un porcentaje bastante bajo de individuos identificó la DM como factor de riesgo cardiovascular, siendo esto consistente con lo encontrado en estudios previos en India $(7,27)$. Este hallazgo resulta inquietante, pues siendo Venezuela un país con una elevada prevalencia de DM en sus distintas formas (28), se esperaba que la mayoría lograra señalar esta condición como un factor predisponte de las ECV. También es necesario hacer notar que, si bien la prevalencia entre sobrepeso u obesidad fue notablemente elevada, menos de la mitad de los participantes lograron identificar al exceso de peso y el sedentarismo como factores de riesgo cardiovascular. Esto guarda similitud con datos reportados en América del Norte y Asia $(6,24)$, evidenciándose la importancia de que los distintos programas educativos enfaticen el rol de la DM y del exceso de peso en el desarrollo de la ECV.

Se espera que al aumentar el nivel de conocimiento sobre los factores de riesgo cardiovascular el individuo modifique sus hábitos en dirección a mantener los distintos parámetros clínicos dentro de los rangos recomendados, por lo que resulta curioso que los participantes de este estudio ubicados en el grupo de alto nivel de conocimiento mostraran mayor frecuencia de alteraciones de casi todos los indicadores clínicos, antropométricos y bioquímicos medidos. Otro estudio ha reportado evidencias similares (29) sugiriendo que una fuente significativa de conocimiento sobre los factores de riesgo cardiovascular se originaría cuando los pacientes acuden a consulta 
médica. El conocimiento, aunque necesario, por sí solo no es un factor determinante para que el individuo tome acciones que contribuyan a mejorar su calidad de vida. Un aspecto importante que pueda contribuir a explicar la asociación directamente proporcional obtenida entre conocimiento y prevalencia de factores de riesgo cardiovascular obtenida en este estudio, lo constituye la llamada estructura del conocimiento, descrita como la forma en que los individuos comprenden la interacción de los factores de riesgo cardiovascular (30). Una persona quizás tenga conocimiento de algún factor de riesgo coronario y se centraliza en prevenir solamente ese factor de riesgo, desconociendo el efecto sinérgico que tienen otros en la aparición de alguna ECV (31).

Finalmente, esta investigación presentó ciertas limitaciones derivadas de la encuesta aplicada, en la cual sólo los participantes evaluados en el año 2009 identificaron los alimentos que predisponen o previenen la aparición de $\mathrm{ECV}$, restringiendo así el número de datos para dicho análisis.

En conclusión, se encontró un porcentaje elevado de individuos con bajo nivel de conocimiento de los factores de riesgo cardiovascular y la frecuencia de alteraciones de los parámetros clínicos, antropométricos y bioquímicos fue mayor entre los participantes con alto nivel de conocimiento de tales factores. Los resultados obtenidos confirman la necesidad de ejecutar estrategias que no sólo eleven el nivel de conocimiento de las comunidades venezolanas, sino también motiven efectivamente a la adopción de un estilo de vida asociado a la reducción de los factores de riesgo cardiovascular y al auto cuidado de la salud

\section{REFERENCIAS}

1. Organización Mundial de la Salud. Informe sobre la Salud en el Mundo 2002: reducir los riesgos y promover una vida sana. Ginebra: Organización Mundial de la Salud; 2002.

2. Elisa FM. The treatment of coronary heart disease: an update. Part 1: an overview of the risk factors for cardiovascular disease. Curr Med Res Opin. 2001; 17:18-26.

3. Ministerio del Poder Popular para la Salud de la República Bolivariana de Venezuela. Anuario de Mortalidad 2006. Caracas: MPPS de Venezuela; 2007.

4. Armitage CJ, Sheeran P, Conner M, et al. Stages of change or changes of stage? Predicting transitions in transtheoretical model stages in relation to healthy food choice. J Consult Clin Psychol. 2004; 72:491-9.

5. Johnston DW, Johnston M, Pollard B, et al. Motivation is not enough: prediction of risk behavior following diagnosis of coronary heart disease from the theory of planned behavior. Health Psychol. 2004; 23:533-8.

6. Potvin L, Richard L, Edwards AC. Knowledge of cardiovascular disease risk factors among the Canadian population: relationships with indicators of socioeconomic status. CMAJ. 2000; 162 Suppl:S5-11. 
7. Saeed O, Gupta V, Dhawan N, Streja L, Shin J, Ku M et al. Knowledge of modifiable risk factors of Coronary atherosclerotic Heart Disease (CASHD) among a sample in India. BMC Int Health Hum Rights. 2009;9:2.

8. Ruiz N, Espinoza M, Triolo M, De Almeida M, Barrios E, Pinto V, et al. Una experiencia de implementación de servicio comunitario en el despistaje de la hipertensión arterial. Salus. 2010; 14(2):41-51.

9. Ruiz N, Espinoza M, Barrios E y Reigosa A. Factores Cardiometabólicos en una Comunidad de Valencia, Venezuela. Rev Salud Pública (Bogotá). 2009; 11(3):383-394.

10. Reynolds T. Declaration of Helsinki revised. J Natl Cancer Inst. 2000; 92:1801-3.

11. Méndez-Castellanos H. Sociedad y Estratificación. Método Graffar-Méndez Castellano. Caracas: Fundacredesa; 1994.

12. Rodríguez-Larralde A, Mijares ME, Nagy E, Espinosa R, Ryder E, et al. Relación entre el nivel socioeconómico y hábitos de vida, con el fibrinógeno y el factor von willebrand en venezolanos sanos y con cardiopatía isquémica. Invest Clin. 2005; 46:157-168.

13. Ferrante D, Virgolini M. Encuesta Nacional de Factores de Riesgo 2005: resultados principales: prevalencia de factores de riesgo de enfermedades cardiovasculares en la Argentina. Rev Argent Cardiol. 2007; 75(1):20-9.

14. Lohman TG, Roche AF, Martorell R. Anthropometric standardization reference manual. Champaign, IL: Human Kinetics Books; 1988.

15. Joint National Committee on Prevention, Detection, Evaluation, and Treatment of High Blood Pressure. The seventh report of the joint national committee on prevention, detection, evaluation, and treatment of high blood pressure. JAMA. 2003; 289:2560-2571.

16. Friedewald WT, Levy RI, Fredrickson DS. Estimation of the concentration of low-density lipoprotein cholesterol in plasma, without use of the preparative ultracentrifuge. Clin Chem. 1972; 18:499-502.

17. World Health Organization. Physical status: the use and interpretation of anthropometry. Report of a WHO Expert Committee. WHO Technical Report Series 854. Geneva: World Health Organization; 1995.

18. Executive summary of the Third Report of The National Cholesterol Education Program (NCEP) Expert Panel on Detection, Evaluation, and Treatment of High Blood Cholesterol In Adults (Adult Treatment Panel III). JAMA. 2001; 285:2486-2497.

19. American Diabetes Association. Screening for type 2 diabetes. Diabetes Care. 2003; 26 Suppl 1: S21-4.

20. Sirit $Y$, Acero C, Bellorin M, Portillo R. Síndrome Metabólico y otros Factores de Riesgo Cardiovascular en Trabajadores de una Planta de Policloruro de Vinilo. Rev Salud Pública (Bogotá). 2008; 10(2): 239-249.

21. Díaz J, Muñoz J, Sierra C. Factores de riesgo para enfermedad cardiovascular en trabajadores de una Institución prestadora de servicios de salud, Colombia. Rev Salud Pública (Bogotá). 2007; 9(1):64-75.

22. Mathieu P, Poirier P, Pibarot P, Lemieux I, Després JP. Visceral obesity: the link among inflammation, hypertension, and cardiovascular disease. Hypertension. 2009; 53(4):577-584

23. Benavides A, Bogantes E, Bolaños M, Arroyo A. Conocimiento de factores de riesgo coronario en la Población de Costa Rica. Rev Costarric Cardiol. 2004; 6:2.

24. Khan M, Jafary F, Jafar T, Faruqui A, Rasool S, Hatcher J et al. Knowledge of modifiable risk factors of heart disease among patients with acute myocardial infarction in Karachim Pakistan: a cross sectional study. BMC Cardiovascular Disorders. 2006; 6:18.

25. Shea S, Stein AD, Basch CE, Lantigua R, Maylahn C, Strogatz DS. Independent associations of educational attainment and ethnicity with behavioral risk factors for cardiovascular disease. Am J Epidemiol. 1991; 134:567-582.

26. Jafary F, Aslam F, Mahmud H, Waheed A, Shakir M, Afzal A, et al. Cardiovascular health Knowledge and behavior in patient attendants at four tertiary care hospitals in Pakistana cause for concern. BMC Public Health. 2005; 5:124. 
27. Murugesan N, Snehalatha C, Shobhana R, Roglic G, Ramachandran A. Awareness about diabetes and its complications in the general and diabetic population in a city in southern India. Diabetes Res Clin Pract 2007; 77:433-7.

28. Avilán JM. Diabetes Mellitus: Epidemiologia de la Diabetes en Venezuela. Gac Méd Caracas. 2004: 112:232-3.

29. Schneider AT, Pancioli AM, Khoury JC, Rademacher E, Tuchfarber A, Miller R et al. Trends in community knowledge of the warning signs and risk factors for stroke. JAMA. 2003; 289:343-6.

30. Lynch EB, Medin DL. Explanatory models of illness: a study of within-culture variation. Cogn Psychol. 2006; 53:285-309.

31. Whitehead D, Russell G. How effective are health education programmes-resistance, reactance, rationality and risk?. Recommendations for effective practice. Int $\mathrm{J}$ Nurs Stud. 2004; 41:163-172. 